\title{
La formación de la competencia responsabilidad social en estudiantes de Ingeniería Química
}

\author{
Alonso Gómez Pérez ${ }^{a}$, Raúl Ortiz Pérez ${ }^{b}$ María de los Angeles Legañoa Ferrác \\ aUniversidad de Camagüey Ignacio Agramonte Loynaz, Cuba, alonso.gomez@reduc.edu.cu \\ bUniversidad de Camagüey Ignacio Agramonte Loynaz, Cuba, raul.ortiz@reduc.edu.cu \\ 'Universidad de Camagüey Ignacio Agramonte Loynaz, Cuba, maria.leganoa@reduc.edu.cu
}

\section{Abstract}

The objective of the work was to determine the main transformations experienced on social responsibility by Chemical Engineering students from the University of Camagüey (UC), as a result of receiving the influence of a pedagogical strategy designed to form the Social Responsibility competence. From the epistemological analysis carried out, the competence of social responsibility for Chemical Engineering students was formulated and described (CSRCES) and a pedagogical strategy for its formation was elaborated. For the analysis of the effects of the application of the abovementioned strategy, a quasi-experimental investigative approach of a longitudinal nature was used, where the collection of pertinent data was carried out by means of questionnaires, the observation by teachers of the students' performance and the analysis of the content of the technical reports delivered by the students who received the influence of the aforementioned strategy during the stage in which they performed the Investigative Practical Training in the first year of their majoring. The results obtained, analyzed by qualitative and quantitative-statistical techniques, supported the recognition of an increase in the Chemical Engineering students' social responsibility in their professional training, commitment for the preservation of the physical environment and social intervention with citizen commitment.

Keywords: training, competence, strategy, social responsibility, engineering, preservation of the environment, citizen commitment

\section{Resumen}

El objetivo del trabajo consistió en valorar las principales transformaciones experimentadas en términos de responsabilidad social, por estudiantes de Ingeniería Química de la Universidad de Camagüey (UC) a consecuencia de recibir la influencia de una estrategia pedagógica diseñada para formar la competencia Responsabilidad Social. A partir del análisis epistemológico realizado se formuló y describió la competencia responsabilidad social para los estudiantes de Ingeniería Química (CRSEIQ) y se elaboró una estrategia pedagógica para su formación. Para el análisis de los efectos de la aplicación de la estrategia conformada se empleó un enfoque investigativo cuasiexperimental y de carácter longitudinal donde la recopilación de los datos pertinentes se efectuó por medio de cuestionarios, de la observación de 
desempeños de los estudiantes efectuada por profesores y, del análisis del contenido de los informes técnicos entregados por los estudiantes que recibieron la influencia de la referida estrategia durante la etapa en que realizaron la Práctica Laboral Investigativa correspondiente al primer año de la carrera. Los resultados obtenidos, analizados por vía cualitativa y cuantitativa-estadística, respaldaron el reconocimiento de un incremento de la responsabilidad social de estudiantes de Ingeniería Química, en su formación profesional, compromiso por la preservación del ambiente fisico e intervención social con compromiso ciudadano.

Palabras clave: formación, competencias, estrategia, responsabilidad social, ingeniería, preservación del medio ambiente, compromiso ciudadano.

\section{Introducción}

La responsabilidad social universitaria (RSU) es un tema actual en las agendas de la educación superior a nivel mundial. En la Conferencia Mundial sobre Educación Superior2009 (UNESCO, 2009) en el comunicado final se hizo un llamado a la responsabilidad social de la educación superior para comprender los complejos desafíos actuales y futuros del mundo; y afrontar los problemas polifacéticos con dimensiones sociales, económicas, científicas y culturales. Asimismo, convocó a los centros de educación superior, para que en el desempeño de sus funciones primordiales (investigación, enseñanza y extensión), se centraran en promover la ciudadanía activa, contribuyendo así al desarrollo sostenible.

Desde la aprobación por la Organización de Naciones Unidas en el año 2015 del importante documento conocido como la Agenda 2030 para el Desarrollo Sostenible (Naciones Unidas, 2018), se intensificó el reclamo hacia las universidades que ya se venía expresando en importantes foros (Lotz, 2014), que estas instituciones desarrollaran un rol más relevante en pos del desarrollo de sociedades sostenibles.

En Cuba, el Ministerio de Educación Superior ha asumido este compromiso. Al respecto Miguel Díaz-Canel, expresaba que la universidad debía ser entendida como un entorno político e intelectual de crucial importancia para la consolidación y fortalecimiento de los valores humanos y la responsabilidad ciudadana, como la mayor y principal proveedora de oportunidades de aprendizaje y de generación de nuevos conocimientos al más alto nivel científico, capaz de incrementar el impacto social de la actividad de investigación - desarrollo - innovación y extensión que acomete, vinculada a la sociedad, aprendiendo de ella y creciéndose para influir en su perfeccionamiento y transformación (Díaz-Canel, 2012).

El ministro de Educación Superior de Cuba ha expresado que dentro del compromiso social de las universidades se encuentra la necesidad urgente de implicarse profundamente en el desarrollo sostenible e inclusivo a partir de sus procesos sustantivos, logrando la formación de profesionales competentes y ciudadanos comprometidos con la sociedad (Saborido, 2018). En ese mismo tenor la viceministra Mesa en el discurso de clausura del Congreso Universidad 2018 expresaba la necesidad de formar a través de las universidades una ciudadanía global en torno al desarrollo sostenible. 
La RSU es un modo ético de actuar de las instituciones de educación superior, en su constante interacción con la sociedad, que implica una toma de conciencia de su papel en la misma y que se extiende al conjunto de sus procesos sustantivos, con la participación de toda la comunidad universitaria, y que promueve la producción y difusión del conocimiento como contribución al desarrollo económico y a la solución de problemas sociales en constante interacción con la sociedad, contribuyendo a la formación de profesionales competentes y ciudadanos comprometidos con la misma y con el desarrollo sostenible. En el sentido apuntado investigadores como Navarro et al (2010), Martí-Vilar et al (2011), Naval y RuizCorbella (2012), Ojalvo y Curiel (2015), Martín y Villaviciencio (2015), Vallaeys (2016), profundizan en la participación integrada de la comunidad universitaria y grupos externos de interés, la articulación de los planes de estudios, la investigación, la extensión y los métodos de enseñanza con la solución de los problemas de la sociedad y la formación de la responsabilidad social del estudiante universitario.

La comunidad científica, sobre todo en las ciencias pedagógicas, le asigna una gran importancia a la formación de la responsabilidad social de los estudiantes universitarios (RSEU) y no es casual la realización de un creciente número de investigaciones en esta dirección. En estudios realizados en España, Chile, Perú y Cuba sobre la RSEU, investigadores como Davidovich et al (2005), de la Calle (2010), , Navarro (2012), Ojalvo y Curiel (2015), Vallaeys (2016), entre otros, demuestran la necesidad de formar en los estudiantes universitarios comportamientos socialmente responsables, en el compromiso con los demás y con el entorno, en la responsabilidad ambiental, en el ejercicio profesional del bien común, en la conducta orientada hacia el bien de todos y en la responsabilidad ciudadana.

Al analizar las consideraciones teóricas de los investigadores precitados, es importante señalar que, aunque enfatizan en la necesidad de atender la formación de la RSEU, se observa como línea de trabajo, que las propuestas presentadas no ofrecen una sistematización teórica y metodológica sobre la formación de la misma desde los diferentes procesos sustantivos y la abordan de forma genérica, sin tomar en cuenta las especificidades del objeto de la profesión para la cual se están formando.

Por ejemplo, en el caso de la formación del ingeniero químico, se debe tener en cuenta que los estudiantes deben estar preparados para comprender y atender el hecho de que gran parte del deterioro que sufre actualmente el ambiente se relaciona de algún modo con los químicos. Lo más importante es que el profesional que egresa de esta carrera debe garantizar un trabajo de calidad, sustentable, ético, con responsabilidad social, en los espacios de desarrollo en las que él será un actor importante.

Un estudio realizado sobre la responsabilidad social en la esfera medioambiental de estudiantes de Ingeniería Química de la Universidad de Camagüey Ignacio Agramonte Loynaz (UC), Cuba (Gómez, Ortiz, \& Legañoa, 2017), revela que existen bajos niveles de responsabilidad social ambiental. Además el estudio develó que existen insuficiencias en otras esferas como son el compromiso ante sus deberes académicos y ciudadanos, y la no integración al currículo de las conocimientos, habilidades y actitudes que debe poseer un estudiante formado con responsabilidad social. 
Diversos autores han abordado la RSEU como una competencia al considerar que integra desempeños para la resolución de problemas en donde se interrelacionan el saber ser, el saber convivir, el saber hacer y el saber conocer. En la literatura revisada se rescatan aportaciones valiosas donde se analiza la importancia de formar a los ingenieros en general, y en particular al ingeniero químico, en competencias de responsabilidad social (Galdeano \& Valiente, 2010; Universidad de Salamanca, 2010; Rodríguez, Mena, \& Rubio, 2011; Male, 2013; Pedraza \& al, 2013; Elmore, 2014). Sin embargo, estas propuestas hablan de competencia de responsabilidad social, compromiso con la preservación del medio ambiente, compromiso ciudadano, compromiso ético, etc., pero no definen las mismas y no se precisan cómo lograrlas.

Por todo el análisis anterior los autores develaron la necesidad de elaborar una estrategia pedagógica para la formación de una competencia relativa a la Responsabilidad Social del estudiante de Ingeniería Química.

\section{Objetivo}

El objetivo del trabajo consistió en valorar las principales transformaciones experimentadas en términos de responsabilidad social, por estudiantes de Ingeniería Química de la UC a consecuencia de recibir la influencia de una estrategia pedagógica diseñada para formar la competencia Responsabilidad Social.

\section{Estrategia pedagógica para la formación de la Competencia Responsabilidad Social en Estudiantes de Ingeniería Química (CRSEIQ)}

En la bibliografía consultada no se encontró una definición y estructura de la CRSEIQ, por lo que se hizo necesario su formulación y descripción. Esta se hizo según el Enfoque Socioformativo de Tobón (2013), el cual tiene en cuenta para el desempeño, el objeto conceptual, la finalidad y la condición de referencia. Para la descripción se determinan cuatro componentes: problemas del contexto, competencia, criterios y evidencias de desempeño.

La competencia se formuló de la siguiente forma:

Cumple de manera comprometida el deber social inherente al objeto de su profesión para contribuir a la solución de problemáticas sociales que existen en su contexto, a través de su preparación académica como ingeniero químico, de la preservación del ambiente físico que ejerce y de la intervención en la vida cívica que realiza, estando marcadas sus actuaciones por la profesionalidad, la ética, la solidaridad y el apego a los principios compartidos por su país.

Los criterios de desempeño se agruparon en tres ejes procesuales como se representa en la tabla 1. 
Tabla 1. Ejes procesuales y criterios de desempeño

\begin{tabular}{|c|c|}
\hline $\begin{array}{l}\text { Ejes } \\
\text { procesales }\end{array}$ & Criterios de desempeño \\
\hline $\begin{array}{c}\text { Implicación } \\
\text { personal en } \\
\text { su } \\
\text { formación } \\
\text { profesional }\end{array}$ & $\begin{array}{l}\text { - Cumple los deberes académicos programados en la carrera. } \\
\text { - Cumple los deberes emanados de las organizaciones juveniles a las } \\
\text { - Ine pertenece. } \\
\text { materias primas, energía y agua en la industria química. } \\
\text { - Ejecuta acciones de servicio comunitario que fortalecen su cultura } \\
\text { científico-técnica, laboral, ambiental y cívica. }\end{array}$ \\
\hline $\begin{array}{l}\text { Preservación } \\
\text { del ambiente } \\
\text { físico }\end{array}$ & $\begin{array}{l}\text { - Obra personalmente a favor del ambiente. } \\
\text { - Promueve el cuidado del ambiente en condiscípulos y } \\
\text { conciudadanos. } \\
\text { Propone alternativas de preservación, mitigación o eliminación de } \\
\text { daños al ambiente relativas a procesos químicos en instituciones } \\
\text { estatales y/o en zonas residenciales. } \\
\text { Impulsa (protagonizando o participando) la aplicación práctica de } \\
\text { alternativas de preservación, mitigación o eliminación de daños al } \\
\text { ambiente provocadas por procesos químicos, en comunidades de } \\
\text { instituciones estatales y/o de zonas residenciales. }\end{array}$ \\
\hline $\begin{array}{l}\text { Intervención } \\
\text { social con } \\
\text { compromiso } \\
\text { ciudadano }\end{array}$ & $\begin{array}{l}\text { Defiende los principios de democracia, justicia, igualdad y derecho } \\
\text { de los individuos. } \\
\text { Induce al acatamiento de las normativas sociales establecidas, } \\
\text { incluyendo el uso racional de los recursos. } \\
\text { Ejercita la urbanidad en ámbitos comunitarios y en general dentro de } \\
\text { la sociedad a su alcance. } \\
\text { Impulsa (protagonizando o participando) la resolución de problemas } \\
\text { predominantemente sociales de las comunidades de su contexto, } \\
\text { cuidando de fortalecer la cohesión de tales colectivos humanos. }\end{array}$ \\
\hline
\end{tabular}

Se definieron como evidencias de desempeño: (a) los registros documentales de calificaciones académicas y de evaluaciones de actitud; (b) los informes de trabajosproyectos de curso, o ponencias presentadas a eventos relacionadas con el trabajo científico estudiantil asociado al perfeccionamiento en la industria química, el empleo de materias primas, energía y agua; (c) los informes de propuestas elaboradas en aras de no provocar, reducir o anular impactos negativos al ambiente, a través del adecuado manejo de sustancias, del agua y de la energía, tanto en instituciones estatales como en el ámbito residencial del hombre, (d) los informes, ensayos o registros documentales sobre argumentaciones presentadas en debates, foros, seminarios, reuniones, etc., y relacionados con el análisis de conceptos de relevancia social como democracia, justicia, igualdad, derechos y deberes ciudadanos, etc.; (e) los registros (documentales, fotográficos o audiovisuales) que demostraran la participación activa y protagónica del estudiante en varias situaciones donde haya realizado actuaciones, en la aplicación práctica de alternativas de evitación, mitigación o eliminación de daños al ambiente, o en la resolución de problemáticas de carácter 
predominantemente social en comunidades (estudiantiles, laborales y residenciales) de su contexto.

Se definieron tres niveles de dominio de la competencia, los cuales se reflejan en la tabla 2:

Tabla 2. Niveles de dominio de la competencia

\begin{tabular}{|c|l|}
\hline Receptivo & $\begin{array}{l}\text { Recibe la información y maneja nociones sobre los procesos } \\
\text { (preparación académica, preservación ambiental e intervención } \\
\text { social), registra los problemas, aplica algún procedimiento } \\
\text { rutinario de resolución y, participa en la búsqueda de alternativas } \\
\text { para la mejora social en comunidades de su contexto. }\end{array}$ \\
\hline \multirow{2}{*}{ Resolutivo } & $\begin{array}{l}\text { Comprende la información y domina los conceptos esenciales } \\
\text { sobre los procesos (preparación académica, preservación } \\
\text { ambiental e intervención social), resuelve problemas sencillos en } \\
\text { sus aspectos clave y, protagoniza posturas ejemplarizantes para } \\
\text { la mejora social en comunidades de su contexto. }\end{array}$ \\
\hline Autónomo & $\begin{array}{l}\text { Gestiona la información y domina con profundidad científica los } \\
\text { conceptos esenciales sobre los procesos (preparación académica, } \\
\text { preservación ambiental e intervención social), resuelve } \\
\text { problemas de diversa índole con los elementos necesarios y, } \\
\text { persuade con propuestas de implicación colectiva para la mejora } \\
\text { social en comunidades de su contexto. }\end{array}$ \\
\hline
\end{tabular}

La validación de la competencia propuesta se hizo a través de grupos de especialistas de la carrera de Ingeniería Química y del Centro de Estudios de Ciencias de la Educación de la UC, los cuales aportaron elementos valiosos que permitieron su perfeccionamiento.

En la investigación se concibió la formación de la competencia desde actividades extensionistas integradas al currículo. Esta decisión se basó en que, particularmente, el quehacer extensionista constituye un canal especialmente propicio para formar la CRSEIQ dada las posisbilidades que brinda a la formación de valores, actitudes y actuaciones favorecedoras del cumplimiento del deber social por parte de los estudiantes. Además, es el proceso sustantivo que favorece la interacción social que requiere la formación de cualquier competencia y la explícita relación universidad-sociedad que le es inherente a la extensión universitaria (Alarcón, 2015).

La estrategia pedagógica tuvo como objetivo de favorecer la formación de la competencia responsabilidad social en estudiantes de Ingeniería Química durante las actividades extensionistas con vínculo curricular. Para su estructuración se tomó en cuenta la formación de la competencia por niveles de dominio, correlacionando los mismos con las Prácticas Laborales Investigativas (PLI) del currículo y el subsiguiente accionar en los proyectos educativos de la carrera. Se definieron dos líneas de acción:

- Línea 1: Abarca las actividades extensionistas integradas a la PLI de los estudiantes correspondiente a cada uno de los años de la carrera y evoluciona en correspondencia con el nivel de dominio de la competencia que se prevé formar en cada etapa de PLI. Se 
incluyen acciones dirigidas a: la formación del compromiso estudiantil con su proyecto de PLI; la actuación pro-ambiental en la esfera de los procesos químicos fundamentales asociados a la industria donde realizan la PLI y, a la socio-transformación de las comunidades tanto internas como aledañas a la entidad industrial estudiada

- Línea 2: Comprende actividades extensionistas integradas en las estrategias educativas de los diferentes años académicos y también evoluciona en correspondencia con el nivel de dominio de la competencia previsto a formar en cada año académico. Se incluyen acciones dirigidas a: la formación del compromiso estudiantil con su proyecto académico; la actuación pro-ambiental en la esfera de los procesos químicos presentes en la vida cotidiana y, la socio-transformación a través de proyectos de impacto social en comunidades intrauniversitarias y residenciales aledañas al centro.

Se determinaron que los actores que participarían en la estrategia pedagógica serían: estudiantes de Ingeniería Química, profesores, profesores tutores y tutores de la práctica laboral investigativa, tanto de la carrera como de las entidades laborales donde realizarían las prácticas.

La estrategia se concibió en cuatro fases: una fase de diagnóstico o de caracterización del estado inicial de los sujetos que recibirán la acción de la estrategia, una fase de planificación, una fase de ejecución de las acciones planeadas (acompañada de evaluaciones formativas o sistemáticas que sustentan momentos intermedios de toma de decisiones para continuar con lo planeado o hacer los reajustes tácticos pertinentes) y, una fase de evaluación final o sumativa que permite juzgar, tanto los resultados de la formación en los educandos de la competencia pretendida, como la calidad alcanzada en la implementación completa de la estrategia diseñada.

En esta comunicación se recogen los resultados de la implementación de la estrategia pedagógica durante las PLI.

\section{Implementación de la estrategia pedagógica durante las Prácticas Laborales Investigativas}

La aplicación de la estrategia para la formación de la CRSEIQ, a través de su dirección o línea de acción asociada a las actividades extensionistas integradas a la Práctica Laboral Investigativa que se describe en este apartado, ocurrió en el curso 2018-19 con los 32 estudiantes de primer año de la carrera de Ingeniería Química de la UC. Esta población estudiantil para la etapa de realización de la PLI1 es subdividida en subgrupos asignados a diferentes industrias de procesos químicos de la provincia. La asignación de estudiantes se hace atendiendo a factores tales como: cercanía respecto al sitio de residencia de los estudiantes, cantidad de estudiantes en PLI que cada industria puede admitir, correspondencia entre la complejidad del proceso tecnológico de cada industria y la preparación con que cuentan los estudiantes al momento de realizar la PLI, entre otros.

Dada la complejidad de la evaluación práctica de la responsabilidad social de estudiantes universitarios, se optó por la valoración de la información relativa al desempeño de los estudiantes que fuese obtenida por varias vías tales como: aplicación de cuestionarios de autoevaluación de los estudiantes, observación del desempeño de los estudiantes durante la PLI1 por parte de los tutores que los atendieron (profesores de la UC y profesionales de la 
industria) y revisión por los autores de esta investigación, de los informes entregados por los estudiantes como memoria del Trabajo de Curso desarrollado dentro de la PLI1.

Se elaboró el cuestionario de autoevaluación para aplicárselo a los estudiantes al inicio y al final de la etapa de la PLI1. El cuestionario se conformó con base en las tres dimensiones esenciales (ejes procesuales) de la competencia bajo estudio y, quedó constituido por 12 ítems. Los consultados debían ofrecer respuestas según un clasificador ordinal relativo a la frecuencia de realización de las actuaciones descritas en los ítems, y que contó con las categorías de: casi nunca $(\mathrm{CN})$, a veces $(\mathrm{AV})$, normalmente $(\mathrm{N})$, casi siempre $(\mathrm{CS})$, siempre (S) y no responde (NR).

La versión inicial del cuestionario fue sometida al criterio de un grupo de profesores con experiencia en el tema. El grado de convergencia obtenido entre las opiniones recogidas se consideró como positivo, pues el coeficiente de concordancia de Kendall (Siegel, 1970) para la valoración por los expertos de cada uno de los reactivos analizados tuvo un valor mínimo de 0,85 . A continuación se muestra en la tabla 3 los ítems del cuestionario de autoevaluación y el valor de los mismos, los cuales se ponderanron según el tipo de respuesta.

Tabla 3. Ítems del cuestionario de autoevaluación

\begin{tabular}{|c|c|}
\hline Ítems & Valor \\
\hline Implicación personal en su formación profesional & 30 \\
\hline 1- Cumplo con los deberes académicos programados en la carrera & 8 \\
\hline $\begin{array}{l}\text { 2- Cumplo con los deberes emanados de las organizaciones juveniles a las que } \\
\text { pertenezco }\end{array}$ & 8 \\
\hline $\begin{array}{l}\text { 3- Participo en actividades investigativas relacionadas con el uso en la } \\
\text { industria química de: (a) materias primas y materiales, (b) energía, (c) agua }\end{array}$ & 6 \\
\hline $\begin{array}{l}\text { 4- Participo en acciones comunitarias dentro y fuera de la universidad que } \\
\text { contribuyen a mi preparación: (a) científico-técnica y laboral, (b) } \\
\text { ambiental, (c) ciudadana }\end{array}$ & 8 \\
\hline Preservación del ambiente físico & 40 \\
\hline $\begin{array}{l}\text { 5- Me preocupo por preservar un ambiente sano en el contexto donde me } \\
\text { desenvuelvo (casa, residencia universitaria, universidad) }\end{array}$ & 8 \\
\hline 6- Promuevo el cuidado del ambiente entre mis compañeros y conciudadanos & 8 \\
\hline $\begin{array}{l}\text { 7- Identifico situaciones relacionadas con procesos químicos (materias primas } \\
\text { y materiales, ahorro de energía y uso del agua) que ocasionan un daño al } \\
\text { ambiente en instituciones estatales y/o en zonas residenciales. }\end{array}$ & 12 \\
\hline $\begin{array}{l}\text { 8- Participo en la búsqueda de alternativas de solución a las situaciones } \\
\text { relacionadas con procesos químicos (materias primas y materiales, ahorro de } \\
\text { energía y uso del agua) que ocasionan un daño al ambiente en instituciones } \\
\text { estatales y/o en zonas residenciales }\end{array}$ & 12 \\
\hline Intervención social con compromiso ciudadano & 30 \\
\hline $\begin{array}{l}\text { 9- Me comporto de acuerdo a los principios de democracia, justicia, igualdad y } \\
\text { derecho de los individuos }\end{array}$ & 8 \\
\hline $\begin{array}{l}\text { 10- Promuevo el cumplimiento de las normativas sociales establecidas, } \\
\text { incluyendo el uso racional de los recursos. }\end{array}$ & 6 \\
\hline $\begin{array}{l}\text { 11- Actúo en mis relaciones con las personas, conforme al principio de } \\
\text { solidaridad humana, respeto, y convivencia social }\end{array}$ & \\
\hline
\end{tabular}


12- Participo en la búsqueda de alternativas a problemas predominantemente sociales de las comunidades de mi contexto.

El cuestionario se aplicó a los 32 estudiantes que conformaban la población para que se autoevaluaran en relación a los criterios de desempeño de la competencia. Los resultados obtenidos en por ciento se representan en la siguiente gráfica:

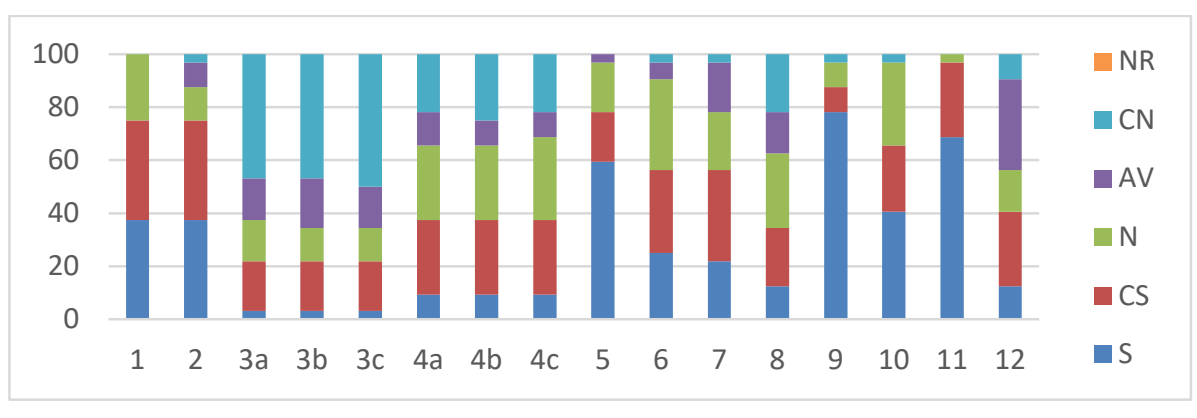

Fig. 1: Diagnóstico inicial en \% por criterios de la CRSEIQ

Se seleccionó una muestra compuesta por siete estudiantes que fueron asignados por la profesora jefa de la asignatura PLI1 para trabajar en tres industrias de procesos químicos (cervezas, helados y recapadora de neumáticos) que por estar ubicadas en el territorio de la ciudad de Camagüey, posibilitaban el seguimiento, tanto por los profesores de la carrera designados como tutores de dichos alumnos, como por los miembros del equipo de investigación encargado de aplicar y evaluar los efectos de la estrategia pedagógica diseñada.

La representatividad de la muestra seleccionada respecto de la población estudiantil del año académico de procedencia fue valorada a través de los resultados del diagnóstico inicial. Se comprobó que los resultados de la muestra estaban correlacionados con los de la población, por lo que se .determinó como adecuada la representatividad de la muestra.

En la etapa de planificación se rediseñaron las guías de las PLI 1 para integrar a las mismas las actividades formativas relacionadas con la competencia. Se desarrollaron en este período talleres con los profesores de la carrera y tutores de las prácticas para prepararlos en relación con la formación de la CRSEIQ.

La ejecución de la estrategia se desarrolló en las entidades designadas. La observación del desempeño de los estudiantes durante la PLI1, fue realizada por los tutores (profesores de la UC y profesionales de la industria) que los atendieron y tuvo como indicadores para la observación los mismos aspectos o criterios de desempeño que se incluyeron en el cuestionario de autoevaluación expuesto en párrafos anteriores. La comparación para cada estudiante, entre la calificación derivada de lo observado por los profesores y la derivada del cuestionario de autoevaluación aplicado al finalizar la PLI1, también constituyó un recurso concebido para contrastar la honestidad y objetividad de las respuestas de los estudiantes en el cuestionario.

La revisión de los informes escritos a modo de Trabajos de Curso correspondientes a lo realizado por los estudiantes durante la PLI1 enfatizó en los aspectos que en mayor medida permitían reflejar el resultado de actuaciones socialmente responsables de los estudiantes. 
Estos fueron: caracterización de la entidad laboral, su comunidad laboral y la comunidad residencial aledaña; descripción de sus principales problemas sociales; análisis de alternativas de solución a los problemas tecnológicos, ambientales y sociales detectados; y contribución a la divulgación de las normativas sociales establecidas y a la participación ciudadana.

\section{Resultados}

A partir de una codificación de las respuestas recogidas de la muestra estudiada por medio del cuestionario de autoevaluación que fue aplicado a la entrada y a la salida de la etapa de la PLI1, se obtuvieron los puntajes totales alcanzados por los estudiantes analizados. En las siguientes gráficas se presentan dichos valores, los cuales a simple vista revelan que para todos los estudiantes de la muestra que recibieron durante la PLI1 la acción de la estrategia pedagógica diseñada, se produce incremento en el puntaje que recoge la contribución de todos los aspectos de la competencia analizada.

La figura 2 representa los resultados del autodiagnóstico inicial expresados en por ciento de la muestra.

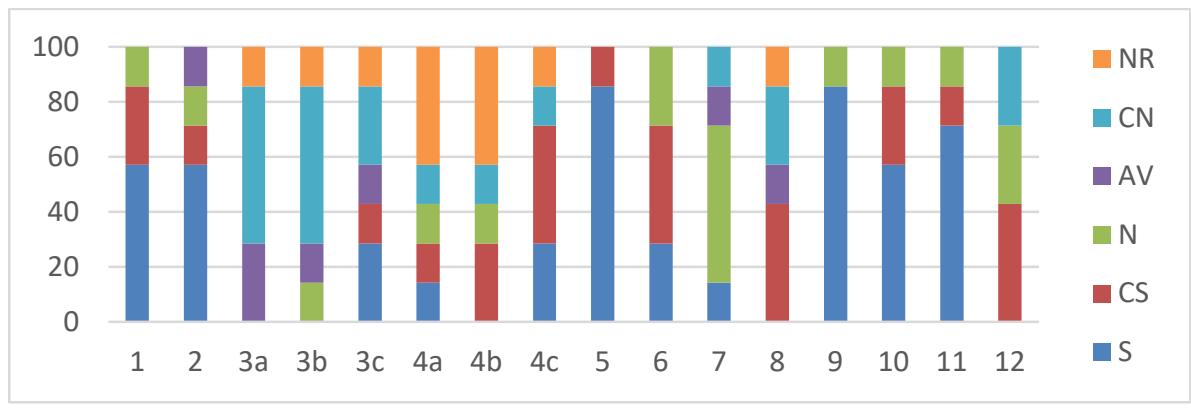

Fig. 2 Autodiagnóstico inicial en \% de la muestra

La figura 3 muestra los resultados del autodiagnóstico final expresados en por ciento de la muestra.

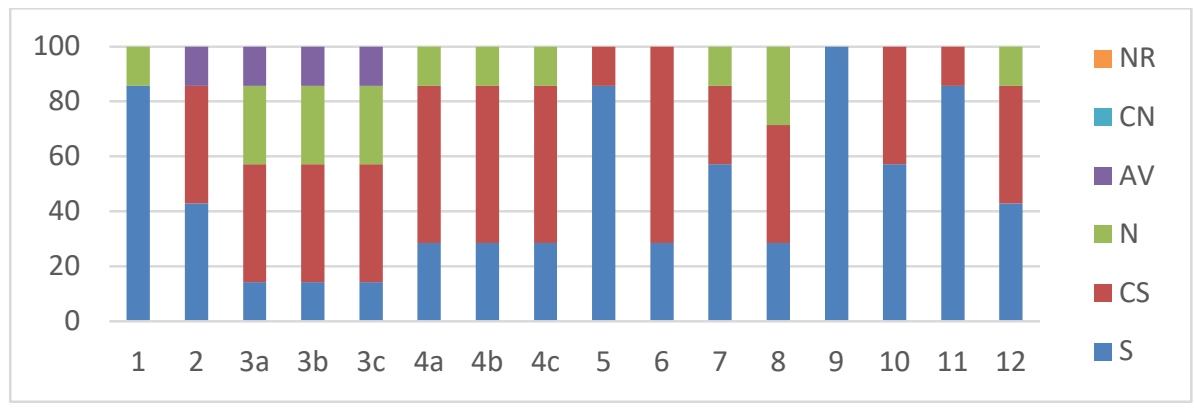

Fig. 3 Autodiagnóstico final en \% de la muestra

Además de lo anterior, y en aras de juzgar la posibilidad de que tal resultado fuese sólo consecuencia del azar, se le aplicó a dicha data la prueba de Wicolxon de rangos con signos para muestras apareadas (Siegel, 1970). Tal prueba, realizada con el programa SPSS, arrojó que el incremento en los puntajes totales reflejados por el cuestionario resulta 
estadísticamente significativo con un nivel de confianza del 95\%, pues la probabilidad de ocurrencia por azar de la distribución de los puntajes analizados es de 0,018 , que es inferior al nivel de significación $(\alpha)$ prefijado para la investigación $(0,05)$.

En la cuarta columna de la Tabla 4 se presenta para cada estudiante de la muestra, la comparación entre los puntajes derivados de la aplicación del cuestionario a la salida de la PLI1, y los derivados de la observación ejecutada por los profesores tutores que atendieron a los referidos estudiantes durante la PLI1. La asociación entre las dos series de valores fue evaluada a través del coeficiente de correlación de rangos de Spearman (Siegel, 1970). El cálculo de este coeficiente con el programa SPSS, reveló que existe una correlación positiva entre las dos series de valores analizadas, que resulta estadísticamente significativa con un nivel de confianza del $95 \%$, pues la probabilidad de ocurrencia por azar de la distribución de los puntajes analizados es de 0,016 lo cual es inferior al nivel de significación $(\alpha)$ prefijado para la investigación $(0,05)$.

Tabla 4. Puntajes derivados del cuestionario y de la observación de los tutores

\begin{tabular}{|c|c|c|c|}
\hline \multicolumn{3}{|c|}{ Puntajes } \\
\hline Estudiante & Antes de la PLI1 & Después de la PLI1 & De la observación de \\
& & & 52 \\
\hline $\mathbf{1}$ & 47 & 56 & 40 \\
$\mathbf{2}$ & 47 & 52 & 56 \\
$\mathbf{3}$ & 52 & 55 & 58 \\
$\mathbf{4}$ & 47 & 58 & 41 \\
$\mathbf{5}$ & 48 & 52 & 42 \\
$\mathbf{6}$ & 34 & 44 & 50 \\
$\mathbf{7}$ & 31 & 53 & \\
\hline
\end{tabular}

Una colección de aspectos importantes extraídos de los informes entregados por los estudiantes como memoria del Trabajo de Curso desarrollado dentro de la PLI1, reveló la manifestación de productos de la actuación estudiantil que son expresiones de transformaciones de la responsabilidad social relacionadas con el compromiso en su formación, la preservación del ambiente físico y la intervención en aspectos ciudadanos especialmente de la comunidad aledaña a la entidad laboral donde desarrollaron la PLI. En estos aspectos también pudieron constatarse nexos con metas de la Agenda 2030.

Se evidenció responsabilidad en los estudiantes por el cumplimiento de las tareas asignadas en la PLI y en la entrega y defensa del Trabajo de curso.

En la primera etapa del informe rendido identificaron las características principales del proceso productivo objeto de estudio; los orígenes e importancia del proceso, el producto y su efecto en la economía y realizaron una caracterización social de la entidad laboral, así como la comunidad aledaña. Indagaron sobre los principales problemas sociales de la comunidad y su vínculo con la entidad laboral.

En la segunda etapa describiron la secuencia de transformaciones fenomenológicas y principales operaciones unitarias que se aplican hasta lograr la obtención de los productos partiendo de las materias primas y analizaron los principales problemas sociales de la entidad laboral y la comunidad aledaña derivados de los procesos químicos estudiados. Este análisis 
lo sustentaron con entrevistas que hicieron a pobladores y directivos, las cuales las grabaron con sus móviles.

La tercera parte del informe estuvo dedicada al análisis y valoración de los procesos estudiados. En esta analizaron si el funcionamiento real del proceso investigado se correspondía con lo normado y se identificaron las principales deficiencias existentes en el funcionamiento del proceso que afectaban la calidad del producto final, los costos de producción o el medio ambiente. Un aspecto novedoso fue el referente a la identificación y formulación de propuestas de eliminación de problemáticas propias de la vida ciudadana dentro de la comunidad representada por los trabajadores de la entidad laboral donde se desarrolla la PLI1 y de la comunidad circundante a la citada entidad laboral y la contribución a la divulgación de las normativas sociales establecidas y la participación ciudadana.

A continuación se reflejan fragmentos de los informes de las PLI que evidencian los aprendizajes en torno a la responsabilidad social.

Liana y Aylen: Realizaron la PLI en la recapadora de neumáticos, al respecto expresaron:

Hace algunos años hubo una reclamación de un vecino, el cual se quejaba de afectaciones que recibía por la contaminación de la fábrica. Debido a esto el ministerio de Ciencia, Innovación, Tecnología y Medio Ambiente (CITMA) paró la producción del proceso durante un período en el cual se realizaron varios estudios, donde se tomaron muestras del aire para ver la contaminación existente en este y se realizaron pruebas de ruido. Se empezaron a tomar medidas para dar solución a estas problemáticas, entre estas se encuentran: elevación de la chimenea, compra de un sistema de extracción de polvo y caldera, se adquirió un compresor nuevo, cerraron todas las ventanas traseras de la entidad con acceso a los patios vecinos. Actualmente no existen estos problemas sociales, pues se siguen empleando medidas para que no exista ningún tipo de vertimiento, y que los desechos sólidos lleguen al destino requerido, no permiten echar al río cercano a la entidad contaminantes y se realizan trabajos voluntarios para mantener limpio sus alrededores.

Yoham y Ana Jéssica: Realizaron la PLI en la fábrica de cervezas, al respecto expresaron:

La entidad presenta grandes edificaciones en muy mal estado, lo que genera problemas que deben solucionarse para evitar posibles daños que puedan afectar a la seguridad de las personas. En la mayoría de los casos la solución óptima es contratar a una empresa o técnicos especializados que se encarguen de realizar un buen plan de mantenimiento periódico. Además, la planta de tratamiento de residuales no está funcionando, lo que genera el vertimiento de sosa caústica en las alcantarillas, pero al estar obstruidas brotan a las calles de la entidad. Sugerimos que en esta zona pongan una señal, que indiquen la existencia de derrames de sosa caútica, hasta que puedan reparar la planta de tratamiento de residuales o contrarrestar la sustancia con un ácido. Por otra parte, la comunidad aledaña tiene sus vías en mal estado, ocasionado por el transporte de la cerveza. La entidad debe atender los problemas de los viales de los vecinos. 
En los informes se constataron manifestaciones de la responsabilidad social de los estudiantes en torno a aspectos tales como: el ahorro y uso racional del agua, de los recursos energéticos y de materiales (especialmente empleados como materias primas de industrias químicas). También en el ámbito del tratamiento y manejo de residuales de la producción, las afectaciones causadas al Medio Ambiente, grado en que se cuida el medio ambiente y dentro de él, a los trabajadores que laboran en el centro.

Vale además destacar que transformaciones que se destacan en la responsabilidad social de los estudiantes, como las antes citadas, no se encontraron en los informes de la PLI1 que fueron entregados por el resto de los estudiantes del mismo año académico a los cuales no se les aplicó la estrategia pedagógica elaborada. y aplicada en esta investigación, para contribuir a la formación de la CRSEIQ.

Por otra parte, un aspecto que permitió valorar positivamente la calidad de la estrategia elaborada, en cuanto a su carácter de recurso para auxiliar el trabajo pedagógico de los profesores que la aplican, fue el que los profesores de la carrera que estuvieron involucrados en su implementación, con prácticamente consenso expresaron conformidad con la capacidad orientadora y organizativa de la estrategia para encauzar el trabajo relacionado con el fomento de actuaciones de responsabilidad social de los estudiantes que aprovechan la cultura del objeto de la profesión de dicho estudiantes.

\section{Conclusiones}

El análisis epistemológico realizado develó que existían falencias en torno a la formación de la responsabilidad social en estudiantes de Ingeniería Química; a su vez, proporcionó un satisfactorio sustento científico para elaborar una estrategia pedagógica orientada a la formación de la competencia responsabilidad social del estudiante de Ingeniería Química (CRSEIQ).

La estrategia pedagógica diseñada en la investigación para contribuir a la formación de la CRSEIQ, en su parte correspondiente al eje que incluye su acción a través de actividades de corte extensionista integradas a la PLI de los estudiantes, resultó satisfactoriamente aplicable a una muestra de estudiantes del primer año de la referida carrera; en tanto los resultados de dicha aplicación que fueron recogidos a través de diferentes vías (cuestionario de autoevaluación de los estudiantes, evaluación otorgada por los profesores que fungieron como tutores durante la PLI1 y análisis por los investigadores del contenido de los informes escritos entregados por los estudiantes como memoria de la PLI1 desarrollada), luego de su análisis cualitativo y también cuantitativo con respaldo de técnicas estadísticas, revelaron adecuados niveles de objetividad y de coherencia entre ellos.

La aplicación de la estrategia pedagógica develó que se produjo una transformación positiva en los estudiantes sujetos de la investigación que se reflejó en tres ámbitos fundamentales: su implicación personal en su formación profesional, su compromiso por la preservación del ambiente físico y su intervención social con compromiso ciudadano. Además, se apreciaron importantes aspectos vinculados a determinadas metas de la Agenda 2030, que denotan el incremento de la responsabilidad social de los estudiantes analizados. 


\section{Referencias}

ALARCÓN, R. (2015). La Extensión Universitaria promotor del cambio y la transformación sociocultural. La Habana: Ministerio de Educación Superior, Cuba.

Davidovich, M., Espina, A., NAVArRo, G., \& SAlazAr, L. (2005). "Construcción y estudio piloto de un cuestionario para evaluar comportamientos socialmente responsables en estudiantes universitarios" en Revista de Psicología, 14, 1, 125-139.

DE LA CALLE, C. (2010). La formación de la responsabilidad social del universitario: un estudio empírico. Madrid: Universidad Complutense de Madrid.

DíAZ-CANEL, M. (2012). "La universidad y el desarrollo sostenible: una visión desde Cuba" en $8 v o$ Congreso de la Educación Superior Universidad 2012. La Habana: M.E.S.

Elmore, B. (2014). "Interanting Community Engagement, Freshman Chemical Engineering and an AIChE Student Chapter" en 12 st ASEE Annual Conference and Exposition. Indianapolis: American Society for Engineering Education.

Galdeano- Bienzobas, C., \& Valiente-Barderas, A. (2010). "Competencias en Ingeniería Química" en Educación Química, 21, 3, 260-264.

Gómez, A., Ortiz, R., \& LegañoA, M. (2017). "Responsabilidad social en la esfera ambiental en Pedagogía Profesional, $15, \quad 3$. $<$ http://www.pedagogiaprofesional.rimed.cu $>$ [Consulta: 24 de septiembre de 2018]

LOTZ, H. (2014). Conferencia Mundial de la UNESCO sobre la Educación para el Desarrollo Sostenible. Aichi-Nagoya, Japón: UNESCO. $<$ https://unesdoc.unesco.org/ark:/48223/pf0000232888_spa $>$ [Consulta: 5 de enero de 2019]

MALE, S. (2013). "Generic Engineering Competences: A Review and Modelling Approcch" en Education Research and Perspective, 37,1, 25-51.

MARTí - VILAR ET AL. (2011). "Responsabilidad Social Universitaria: Estudio iberoamericano sobre influencia de la educación superior en la formación de profesionales responsables con la sociedad" en Técnica Administrativa, 10, 3. $<$ http://www.cyta.com.ar/ta1003/v10n3a1.htm> [Consulta: 10 de diciembre de 2018]

MARTín, E., \& VillaViciencio, V. (2015). "La Responsabilidad Social Universitaria: Retos y Perspectivas" en E. Aponte Hernández La Responsabilidad Social de las Universidades: Implicaciones para América Latina y el Caribe. San Juan, Puerto Rico: UNESCO - IESALC.

NACIONES UnIDAS. (2018). La Agenda 2030 y los Objetivos de Desarrollo Sostenible: una oportunidad para América Latina y el Caribe. Santiago: Naciones Unidas. $<$ https://repositorio.cepal.org/bitstream/handle/11362/40155/24/S1801141_es.pdf> [Consulta: el 5 de enero de 2019] 
Naval, C., \& Ruiz-Corbella, M. (2012). "Aproximación a la Responsabilidad Social Universitaria: La respuesta de la universidad a la sociedad" en Bordón, 64, 3, 103115 .

NAVARRO ET AL. (2010). "Universitarios y Responsabilidad Social" en Calidad en la Educación, 33, diciembre, 101-121.

NAVARRO, G. (2012). Moralidad y Responsabilidad Social: Bases para su desarrollo y educación. Concepción, Chile: Universidad de Concepción. $<$ http://www2.udec.cl/rsu/images/stories/doc/2017/marzo/moralidad_y_responsabi lidad_social_bases_para_su_desarrollo_y_educacion.pdf $>$ [Consulta: el $17 \mathrm{de}$ marzo de 2018]

OJALVO, V., \& CURIEL, L. (2015). "Las formación integral del estudiante y la formación continua de los profesores en la Educación Superior cubana: el papel de la Responsabilidad Social Universitaria (RSU) en su consecución" en Journal of Educational, Cultural and Psychological Studies, 12, 257-282. doi:10.7358/ecps2015-012-ojal

PedrazA, G., \& AL, E. (2013). Educación Superior en América Latina: reflexiones y perspectivas en Quimica. Deusto: Universidad de Deusto, España.

Rodríguez, M., Mena, D., \& Rubio, C. (2011). Competencias que pueden desarrollarse en la Asignatura de Mecánica en el Currículo de Ingeniería en Formación Universitaria, 4, 4, 3-12.

SABORIDO, J. R. (2018). "La Universidad y la Agenda 2030 de Desarrollo Sostenible en el centenario de la Reforma de Córdoba. Visión desde Cuba" en 11no Congreso de la Educación Superior Universidad 2018, La Habana: MES.

SIEGEL, S. (1970). Diseño experimental no paramétrico. La Habana: Ed. Revolucionaria.

TовÓn, S. (2013). Formación integral y competencias: pensamiento complejo, currículo, didáctica y evaluación. Bogotá: Ecoe.

UNESCO. (2009). Conferencia Mundial sobre la Educación Superior-2009: la nueva dinámica de la educación superior y la investigación para el cambio social y el desarrollo. París: UNESCO. $<$ http://www.unesco.org/education/WCHE2009/comunicado_es.pdf $>$ [Consulta: 24 de septiembre de 2018]

Universidad de SAlamanCA. (2010). Grado de Ingeniería Química. Facultad de Ciencias Quimicas. Competencias. Salamanca: Universidad de Salamanca.

Vallaeys, F. (2016). Introduccción a la Responsabilidad Social Universitaria (Vol. 6). Barranquilla, Colombia: Universidad Simón Bolívar. 